\title{
Defective Escape Behavior in DEAH-Box RNA Helicase Mutants Improved by Restoring Glycine Receptor Expression
}

\author{
Hiromi Hirata, ${ }^{1,2,3}$ Kazutoyo Ogino, ${ }^{1}$ Kenta Yamada, ${ }^{1}$ Sophie Leacock, ${ }^{4}$ and Robert J. Harvey ${ }^{4}$ \\ ${ }^{1}$ Center for Frontier Research, National Institute of Genetics, Mishima 411-8540, Japan, ${ }^{2}$ Department of Genetics, Graduate University for Advanced Studies \\ (SOKENDAI), Mishima 411-8540, Japan, ${ }^{3}$ PRESTO, Japan Science and Technology Agency, Osaka 567-0047, Japan, and ${ }^{4}$ Department of Pharmacology, UCL \\ School of Pharmacy, London WC1N 1AX, United Kingdom
}

RNA helicases regulate RNA metabolism, but their substrate specificity and in vivo function remain largely unknown. We isolated spontaneous mutant zebrafish that exhibit an abnormal dorsal bend at the beginning of tactile-evoked escape swimming. Similar behavioral defects were observed in zebrafish embryos treated with strychnine, which blocks glycine receptors (GlyRs), suggesting that the abnormal motor response in mutants may be attributable to a deficit in glycinergic synaptic transmission. We identified a missense mutation in the gene encoding RNA helicase Dhx37. In Dhx37 mutants, ribosomal RNA levels were unchanged, whereas GlyR $\alpha 1, \alpha 3$, and $\alpha 4$ a subunit mRNA levels were decreased due to a splicing defect. We found that Dhx37 can interact with GlyR $\alpha 1, \alpha 3$, and $\alpha 4$ a transcripts but not with the GlyR $\alpha 2$ subunit mRNA. Overexpression of GlyR $\alpha 1, \alpha 3$, or $\alpha 4$ a subunits in Dhx37-deficient embryos restored normal behavior. Conversely, antisense-mediated knockdown of multiple GlyR $\alpha$ subunits in wild-type embryos was required to recapitulate the Dhx37 mutant phenotype. These results indicate that Dhx37 is specifically required for the biogenesis of a subset of GlyR $\alpha$ subunit mRNAs, thereby regulating glycinergic synaptic transmission and associated motor behaviors. To our knowledge, this is the first identification of pathologically relevant substrates for an RNA helicase.

\section{Introduction}

RNA helicases belong to a large DNA/RNA helicase superfamily and are classified into Upf1-like, Ski2-like, RIG-I-like, NS3/ NPH-II, DEAD-box, and DEAH-box subfamilies (Bleichert and Baserga, 2007). These RNA helicases take part in various aspects of RNA metabolism. In yeast, the DEAH-box protein Dhr1p regulates the synthesis of ribosomal RNA (rRNA) and is involved in the preribosomal complex (Colley et al., 2000; Grandi et al., 2002). However, the biological function of the vertebrate homolog Dhx37 has not been investigated. In vivo physiological functions of RNA helicases remain largely unknown, with the exception of a few involved in disease (Moreira et al., 2004; Boon et al., 2007; Pena et al., 2007). Preference for certain substrate RNAs was reported only for RNA helicase A (Hartman et al., 2006). Whether other RNA helicases have specific targets remains unknown.

\footnotetext{
Received March 17, 2013; revised July 2, 2013; accepted July 19, 2013.

Author contributions: H.H. designed research; H.H., K.O., K.Y., and S.L. performed research; H.H., K.O., K.Y., and S.L. analyzed data; H.H. and R.J.H. wrote the paper.

This work was supported by Grants-in-Aid for Scientific Research (23115719 and 25920008) from the Ministry of Education, Culture, Sports, Science, and Technology of Japan, and by Takeda Science Foundation to H.H.; and by Action Medical Research (1966) and the Medical Research Council (G0500833, G0601585 and J004049) to R.J.H. We thank Megumi Takahashi, and Drs. Toshinobu Fujiwara, Norimasa Iwanami, Masashi Tanimoto, Shin Takagi, Yoichi Oda, Koichi Kawakami, Sean E. Low, and John Y. Kuwada for preliminary findings, materials, and discussion.

The authors declare no competing financial interests.

This article is freely available online through the J Neurosci Author Open Choice option.

Correspondence should be addressed to Hiromi Hirata. Center for Frontier Research, National Institute of Genet-

ics, 1111 Yata, Mishima 411-8540, Japan. E-mail: hihirata@nig.ac.jp.

DOI:10.1523/JNEUROSCI.1157-13.2013

Copyright $\odot 2013$ Hirata et al.

This is an Open Access article distributed under the terms of the Creative Commons Attribution License (http://creativecommons.org/licenses/by/3.0), which permits unrestricted use, distribution and reproduction in any medium provided that the original work is properly attributed.
}

Zebrafish represent a useful model for motor study. At $2 \mathrm{~d}$ postfertilization (dpf), tactile stimulation evokes an escape behavior, which consists of an initial turn and subsequent swimming (Saint-Amant and Drapeau, 1998). Forward genetics has identified zebrafish mutants as being defective in this escape behavior (Granato et al., 1996). For example, bandoneon mutants carry mutations in $g l r b b$, encoding the glycine receptor (GlyR) $\beta$ b subunit. Since GlyR $\alpha$ and $\beta$ subunits form heteromeric pentamers, with the $\beta$ subunit being essential for the synaptic clustering of GlyRs (Lynch, 2004), the GlyR $\beta$ b mutants exhibited a loss of inhibitory glycinergic synaptic transmission. Due to the consequent loss of the reciprocal inhibition between the left and right sides of the spinal cord, motor neurons activate simultaneously on both sides (Grillner, 2003; Fetcho et al., 2008), which results in bilateral muscle activation and thus dorsal flexure of the body in GlyR $\beta \mathrm{b}$ mutants (Hirata et al., 2005). The same motor deficit is observed in zebrafish embryos treated with a high concentration of strychnine, a GlyR antagonist (Granato et al., 1996; Hirata et al., 2005; McDearmid et al., 2006). On the other hand, the application of strychnine at a lower concentration causes a transient dorsal bend followed by swimming.

In this study, we characterized a zebrafish mutant, $d h \times 37^{\text {nig1 }}$, that exhibits a tactile-evoked dorsal bend, followed by swimming. Positional cloning revealed a missense mutation in the DEAHbox RNA helicase Dhx37. In $d h \times 37^{\text {nigl }}$ mutants, mRNA levels of selected GlyR $\alpha$ subunit genes were decreased, whereas rRNA levels were unchanged. Indeed, our RNA analyses showed that Dhx37 binds to a subset of GlyR transcripts and regulates their 


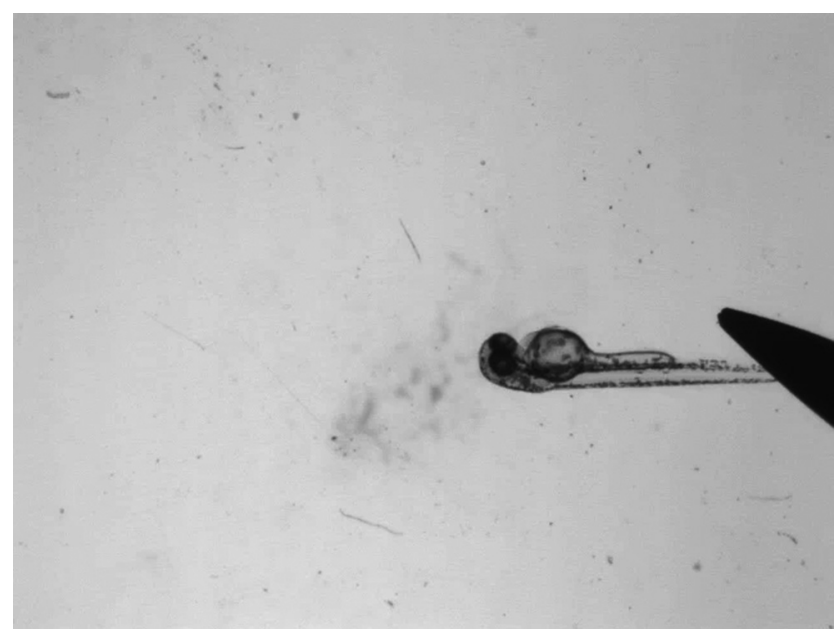

Movie 1. Touch-induced escape behavior at $2 \mathrm{dpf}$. Clip 1, Tactile stimulation of wild-type zebrafish embryo via the tail induced an escape response. Clip 2, Most nig 1 mutants responded to touch with a dorsal bend of the body followed by escape swimming. Clip 3, Some of the nig 1 mutants exhibited a strong dorsal bend without subsequent swimming. Clip 4, Level 1 (normal), exhibiting a lateral turn and subsequent burst swimming. Clip 5, Level 2 (mild), Exhibiting a bend to the dorsal side instead of a lateral turn followed by swimming of $>2 \mathrm{~cm}$. Clip 6, Level 3 (severe), Exhibiting a dorsal bend without escape swimming. Clip 7, Some NFPS-injected mutants responded to touch without dorsal bend at the onset of the escape.

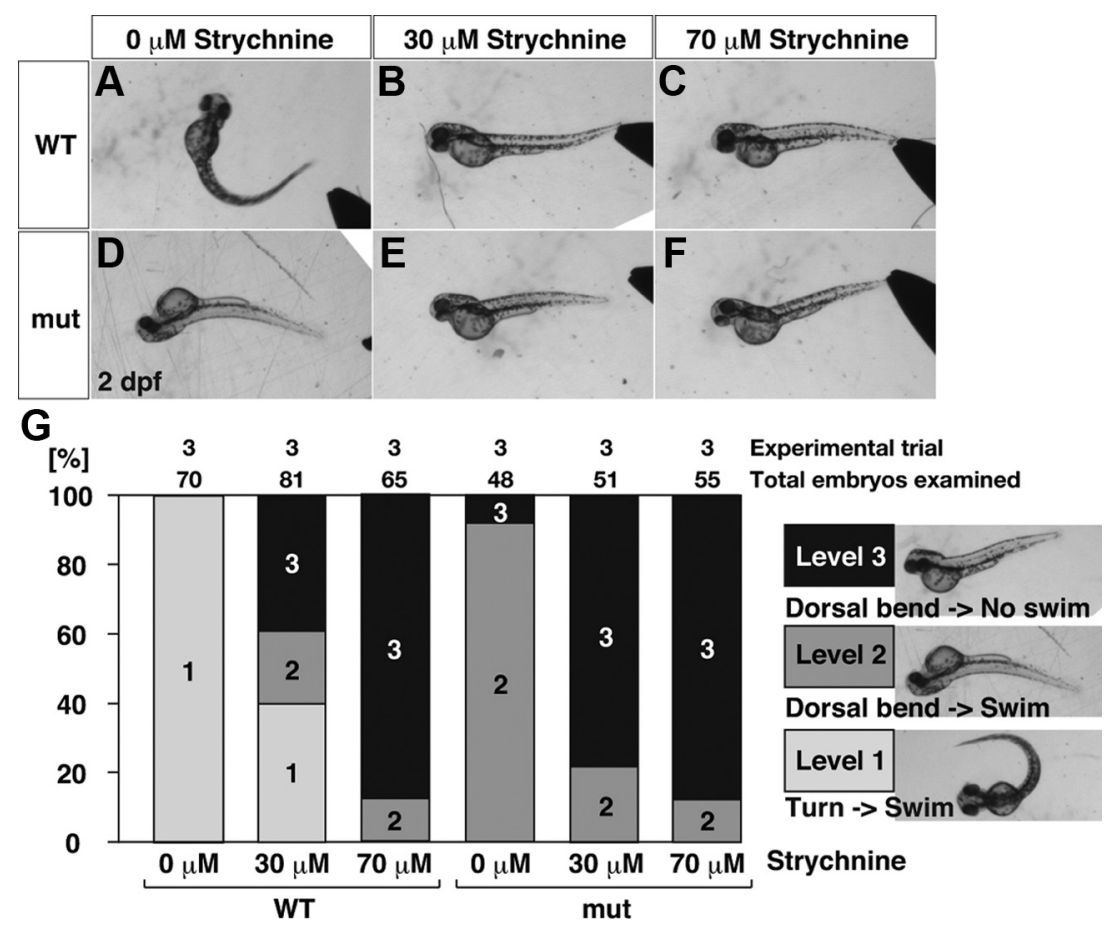

Figure 1. Zebrafish nig1 mutants display a dorsal bend in a tactile-evoked escape behavior. $A$, At 2 dpf, wild-type embryos showed a lateral turn (level 1). $\boldsymbol{B}$, In the presence of $30 \mu \mathrm{m}$ strychnine, some wild-type embryos exhibited a dorsal bend followed by swimming (level 2). $C$, In the presence of $70 \mu \mathrm{m}$ strychnine, most wild-type embryos displayed a dorsal bend without subsequent swimming (level 3). $\boldsymbol{D}$, Mutant embryos responded to touch with dorsal flexure of the body followed by swimming. $\boldsymbol{E}, \boldsymbol{F}, \mathrm{In}$ the presence of 30 and $70 \mu \mathrm{m}$ strychnine, mutants responded to tactile stimulation with a dorsal bend; this was sometimes but not usually followed by swimming. $\mathbf{G}$, The histogram represents the ratio of embryos exhibiting level 1, 2, or 3 .

splicing. Overexpression of selected recombinant GlyR $\alpha$ subunits restored the normal touch response in Dhx37-depleted embryos. Thus, Dhx37 plays an essential role in the biogenesis of GlyR $\alpha$ subunit mRNAs and is indispensable for normal escape behavior in vertebrates.

\section{Materials and Methods}

Animals. Zebrafish were bred and raised according to the guidelines set forth by the National Institute of Genetics. The $d h x 37^{\text {nig1 }}$ mutation was found in zebrafish breeding stock obtained from a pet shop. Animals from either sex were used in this study.

Video recording. Embryonic behaviors were recorded using a highspeed camera at 200 frames per second (HAS-220, Ditect), as previously described (Hirata et al., 2005).

Electrophysiology. The dissection protocols for in vivo patch recordings have been described previously (Hirata et al., 2005). To record spontaneous glycinergic currents, $1 \mu \mathrm{M}$ tetrodotoxin (TTX; Sigma), $10 \mu \mathrm{M}$ 6-cyano-7-nitroquinoxaline-2,3-dione (CNQX; Sigma), $50 \mu \mathrm{M}$ aminophosphonovalerate (APV; Sigma), $10 \mu \mathrm{m}$ bicuculline (Sigma), and $20 \mu \mathrm{M}$ D-tubocurarine (Sigma) were bath applied.

Mapping, cloning, mRNA rescue, and antisense knockdown. A mutant carrier fish was crossed with a WIK fish for meiotic mapping. Cloning, mRNA rescue, and antisense knockdown were performed as described previously (Hirata et al., 2005). The following primers and morpholino oligonucleotides (MOs) were used. We performed injection of MOs and/or RNAs in three independent experimental trials and obtained consistent results: zDhx37: ATGGGCAGATTGAGAAAGAAACA, TTAAAAGTCTAGGTTT AGAAGGTCAGA; Dhx37 MO1: TGTTTCTTTCTCAATCTGCCCATGG; Dhx37 MO2: ATCAAGTGTTTTACCTTGTTGCGGA; GlyR $\alpha 1$ MO: AC AAATAAATCCCGAGTGCGAACAT; GlyR $\alpha 2$ MO: GGAGTTTGACCG AAGGGCGAGTCAT; GlyR $\alpha 3$ MO: CAGAGCGCAGTCTTTTCCGAGT CAT; GlyR $\alpha 4$ a MO: AAATCCTTATGACCTGAGGGAGCAT; GlyR $\alpha 4 \mathrm{~b}$ MO: C GATCCTCCAGATCACAGAAAACAT; GlyR $\beta$ a MO: G TGCTGACTTACGAGGGACA GATGA; GlyR $\beta$ bMO:AAATGCACTTACGAAG GACAGTAA; and control MO (random sequence with no target): CCTCTTACCTCAGTTACAAT TTATA.

To pharmacologically increase glycinergic transmission for behavioral rescue, NFPS $(N[3-$ (4'-fluorophenyl)-3-(4'-phenylphenoxy)propyl] sarcosine, Santa Cruz Biotechnology), a blocker of Gly transporter 1 (GlyT1), was injected into mutants as described previously (Cui et al., 2005).

RNA analysis. Northern blotting was performed using a full coding sequence as a probe. Quantitative PCR (qPCR) was performed using a KAPA SYBR Fast qPCR Kit (Kapa Biosystems). The following PCR primers were used: GlyR $\alpha 1$ : CTCTCTTCCCAAGGTCTCG, GCCTCGTCCT CCTTCAG; GlyR $\alpha 2$ : CTGTACAGCATCAGGC TGAC, TGGTGTATCCGAAGCTCTCC; GlyR $\alpha 3$ : GCAGCTGGAGAGTTTTGGTTAC, GCAT GTGAACTTGCCTGTGTTG; GlyR $\alpha 4$ a: GAAT GTGCTTTACAGCATCAGGC, CGTTCATGGT GTAGCCAAAGC; GlyR $\alpha 4$ b: GTATAGCATC AGACTCACGCTG,CAGATCATTCATAGTG TAGCCAAAGC; GlyR $\beta$ a: CGGCCGAATTTC AAAGGAATC, GCAGGAAGATATTCACAC GATAATCCATT;GlyR $\beta$ b:GTTCTCATCAGC ATGAGGTTGTC, GTCATCTGTGGTGTAA CCAAAGC; Gephyrin a: GAGTGTTTATGA AGCCAGGTTTGC, CTGACACTGGATTACC TGGAAG; Gephyrin b: GGATGCTCTCCAGA CCTG, GATGAACTGGAAGCATTCCTGTG; GlyT1: CTACAGAAATGGAGGAGGTGC, CA TTCCATAGCCCACACCTTTAAAC; GlyT2: GGTCGGAATCTCTTACATTTATGG, GTAC AGACTAAGTGCCAAAATGAATGTG; vesicular inhibitory amino acid transporter (VIAAT): AACGCGATCCAGGGCATG, GAGGATTTTGCCTGTGTA GCAG; and Actb1: CCTTCCTTCCTGGGTATGG, GGGGGAGCAATG ATCTTGATC.

Immunostaining. Immunostaining of spinal cord sections was performed as described previously (Hirata et al., 2005). The following anti- 
bodies were used: anti-GlyR $\alpha$ (1:1000; mAb4a, $\mathrm{IgG}_{1}$, Synaptic Systems); anti-gephyrin (1: 1000 ; clone $45, \operatorname{IgG}_{1}$, Synaptic Systems); antiacetyl tubulin (1:1000; 6-11B-1, $\operatorname{IgG}_{2 \mathrm{~b}}$, Sigma); Alexa Fluor 488-conjugated anti-mouse $\mathrm{IgG}_{1}$; Alexa Fluor 488-conjugated anti-mouse $\operatorname{IgG}_{2 \mathrm{~b}}$ (1:1000; Invitrogen). Quantitative analysis of immunolabeling signals was done using ImageJ (NIH).

Immunoprecipitation and Western blotting. Immunoprecipitation and Western blots were performed as described previously (Hirata et al., 2005). Anti-FLAG affinity gel and mouse IgG agarose (Sigma) were used for immuneprecipitation and its control. Anti-GlyR $\alpha$ (1: 2000), anti-gephyrin (1:2000), anti-acetyl tubulin (1:2000), anti-FLAG (1:1000; M2, Sigma), and peroxidase-conjugated antimouse IgG (1:1000; Invitrogen) were used in Can Get Signal immunoreaction enhancer solution (Toyobo). The intensity of bands was quantified using Multi Gauge (Fujifilm).

\section{Results}

$d h \times 37^{\text {nig1 }}$ mutants display abnormal escape behavior due to a defect in glycinergic synaptic transmission A recessive mutation, nig1 (later referred to as $d h \times 37^{\text {nigl } 1}$ ), was identified in our breeding stock of zebrafish. At $2 \mathrm{dpf}$, tactile stimulation of wild-type zebrafish induced an escape response that consists of an initial turn to the lateral side and subsequent swimming (Movie 1, Clip 1). The initial turn is executed by activation of the trunk muscles on one side, and the subsequent swimming is mediated by alternating muscle contractions on either side of the trunk. By contrast, most nig1 mutants (92\%, 44/48 mutants) responded to touch with a dorsal bend of the body followed by swimming (Movie 1, Clip 2). The rest of the mutants ( $8 \%, 4 / 48$ mutants) exhibited a strong dorsal bend without subsequent swimming (Movie 1, Clip 3). Since a dorsal flexure of the trunk is typically seen in embryos treated with strychnine, a GlyR antagonist, we assayed the touch response following exposure to a low or high dose of strychnine in the bath and classified the response into the following three groups:

level 1 (normal), exhibiting a lateral turn and subsequent swimming (Movie 1, Clip 4); level 2 (mild), exhibiting a dorsal bend followed by swimming of $>2 \mathrm{~cm}$ (Movie 1, Clip 5); and level 3 (severe), exhibiting a dorsal bend without escape swimming $(<2$ $\mathrm{cm}$; Movie 1, Clip 6). In the absence of strychnine, all of the wild-type embryos exhibited a normal response (Fig. 1A, G). Application of $30 \mu \mathrm{M}$ strychnine induced level $2(21 \%, 17 / 81)$ and level $3(38 \%, 31 / 81)$ responses in wild-type embryos (Fig. 1B). This strychnine-induced motor deficit was dose dependent, because treatment with $70 \mu \mathrm{M}$ strychnine worsened the response (level 3: 88\%, 57/65; Fig. 1C). Without strychnine, most mutant embryos exhibited the level 2 phenotype (Fig. 1D). Treatment with $30 \mu \mathrm{M}$ strychnine induced severe motor deficits (level 3: $78 \%, 40 / 51$; Fig. $1 E$ ), as did treatment with $70 \mu \mathrm{M}$ strychnine
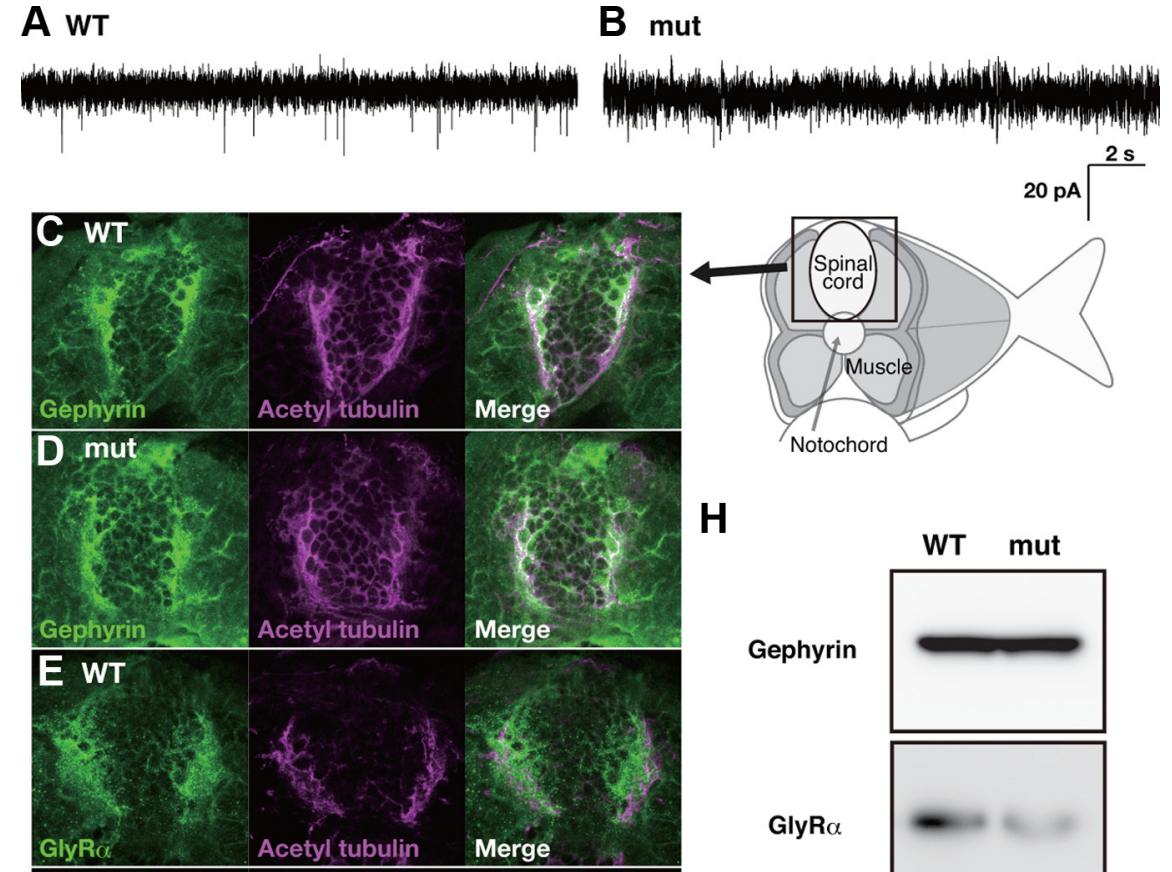

H
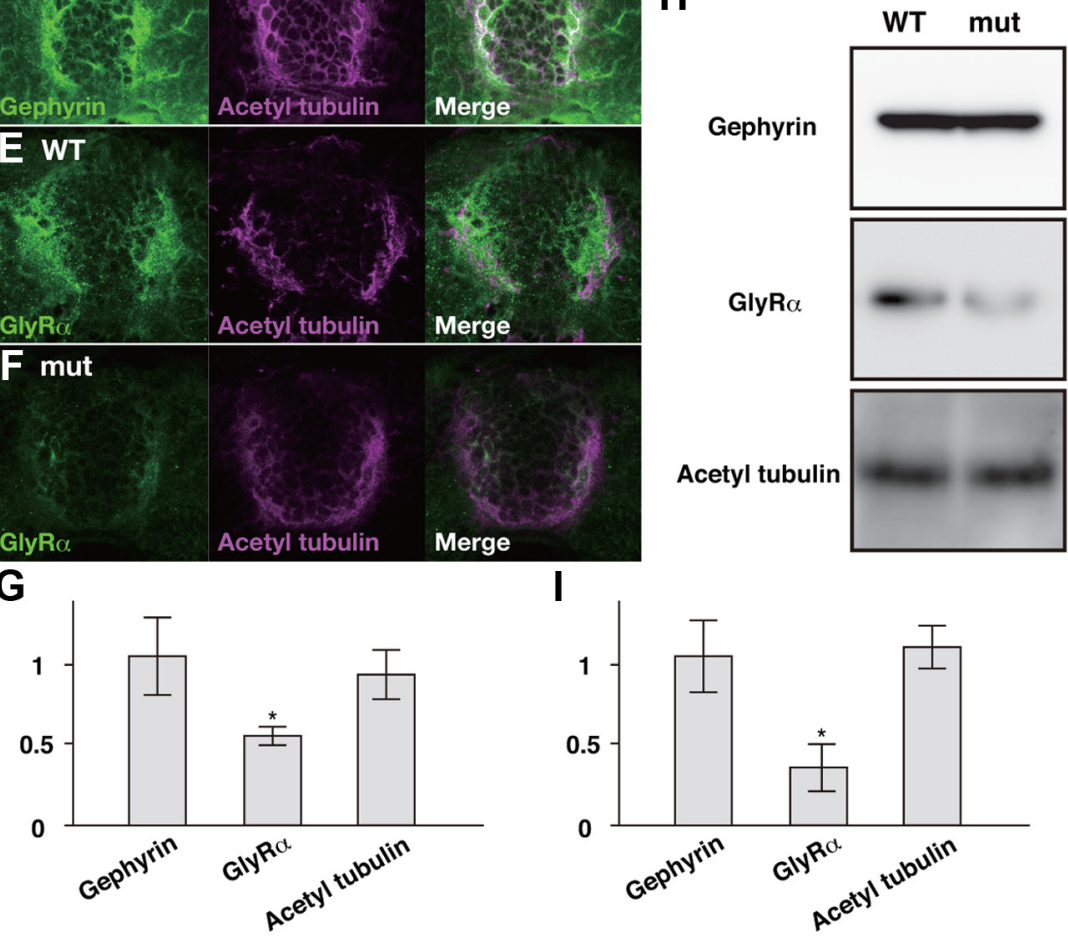

Figure 2. Glycinergic synaptic transmission is affected in nig 1 mutants. $\boldsymbol{A}$, Spontaneous glycinergic synaptic currents recorded in a wild-type motor neuron following blockade of $\mathrm{Na}^{+}$channel and AMPA, NMDA, GABA ${ }_{A}$, and $\mathrm{nACh}$ receptors with TTX, CNQX, APV, bicuculline, and D-tubocurarine, respectively. $\boldsymbol{B}$, The frequency of spontaneous events is decreased in nig1 mutants. $\boldsymbol{C}$, Anti-GlyR $\alpha$-labeled clusters of GlyRs at glycinergic synapses. $\boldsymbol{F}$, GlyR signal was reduced in the mutant spinal cord. $\boldsymbol{G}$, showing the intensity of immunolabeling signals in mutants compared with wild-type fish. $\boldsymbol{H}$, Western blots probed with anti-GlyR $\alpha$ showed the reduction of GlyR protein in mutants. Protein levels of gephyrin and acetylated tubulin were unaffected in mutants. I, Histograms showing the intensity of bands in mutants compared with wild-type fish.

(level 3: 87\%, 48/55; Fig. $1 F$ ). These results suggest that glycinergic transmission is compromised in nig1 mutants. Although nig1 mutants did not show apparent developmental defects, they became thinner and died at 7-10 dpf, possibly from an ineffective motor response and feeding difficulties.

To see whether glycinergic transmission is affected in nig1 mutants, we measured miniature glycinergic currents in motor neurons at $2 \mathrm{dpf}$. In wild-type zebrafish, spontaneous glycinergic synaptic currents in the presence of TTX, CNQX, APV, bicuculline, and D-tubocurarine, which block $\mathrm{Na}^{+}$channel, AMPA, $\mathrm{NMDA}, \mathrm{GABA}_{\mathrm{A}}$, and $\mathrm{nACh}$ receptors, respectively, were observed at $0.39 \pm 0.17 \mathrm{~Hz}(n=7$; Fig. $2 A)$, with a frequency comparable to a previous report $(0.33 \pm 0.07 \mathrm{~Hz}$; Hirata et al., 2005). However, the spontaneous events were less frequently seen 


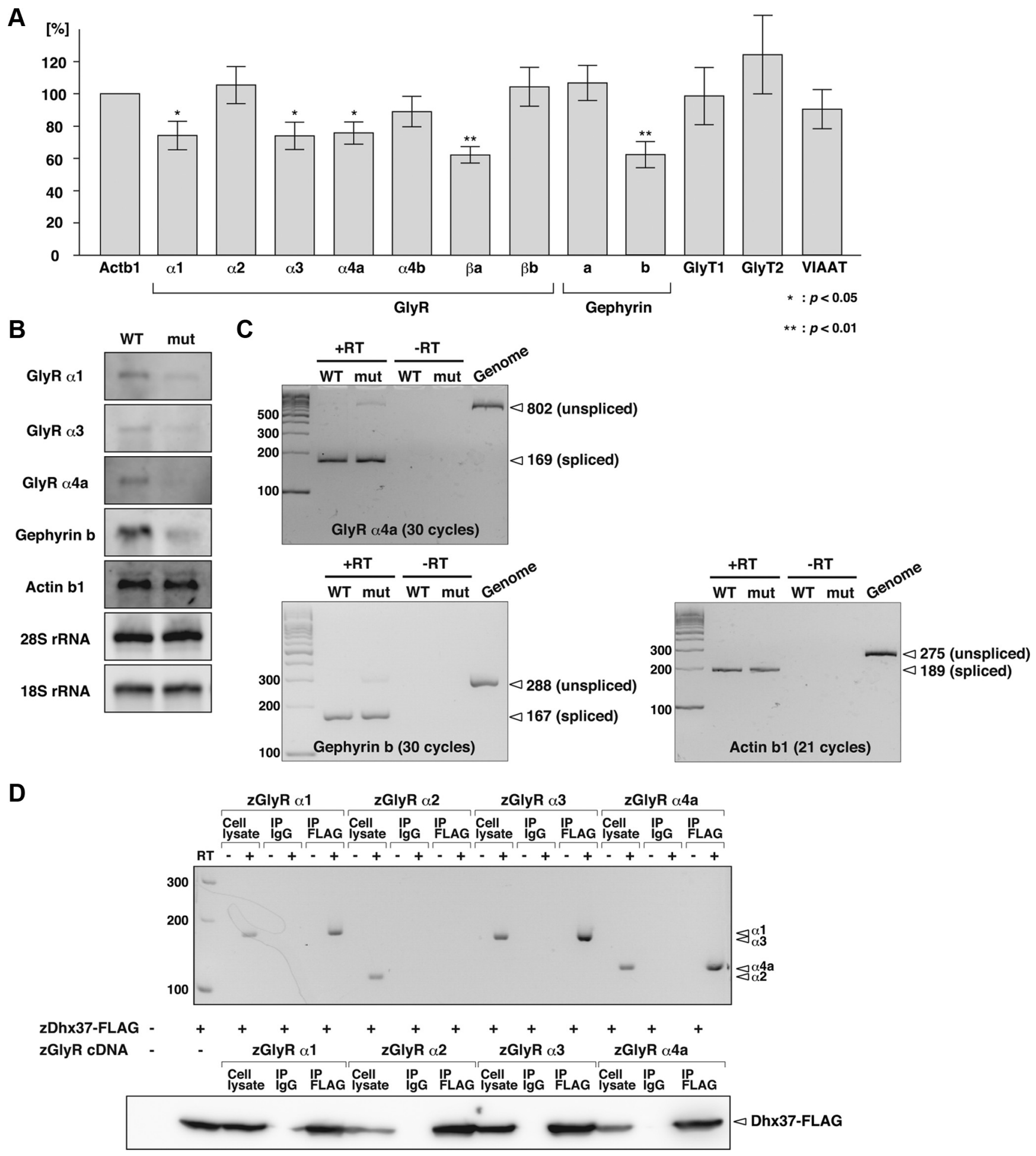

Figure 3. mRNA levels for GlyR $\alpha 1, \alpha 3$, and $\alpha 4$ a subunits are decreased in $d h \times 37^{\text {nig1 }}$ mutants. $\boldsymbol{A}$, The graph represents the ratio of mutant transcripts compared with wild-type fish estimated by qPCR. $\boldsymbol{B}$, Northern blots of total RNA. Note that mRNA levels of GlyR $\alpha 1, \alpha 3$, and $\alpha 4$ a subunit and gephyrin b decreased in mutants. $\boldsymbol{C}$, RT-PCRs to detect unspliced products in mutant transcripts. Unspliced transcripts for GlyR $\alpha 4$ a and gephyrin b were retained in mutants. Genome lanes refer to the validation of unspliced products by genomic PCR. + RT, With reverse transcription; - RT, without reverse transcription. D, HEK293 cells were transfected with GlyR cDNAs and Dhx37-FLAG expression vector. Cell extracts were subjected to immuneprecipitation with anti-FLAG, followed by RT-PCR. Transcripts for GlyR $\alpha 1, \alpha 3$, and $\alpha 4$ a subunits but not of $\alpha 2$ subunits were coprecipitated with Dhx37-FLAG. The presence of Dhx37-FLAG in the immunoprecipitates was verified by Western blotting with an anti-FLAG.

in mutants $(0.08 \pm 0.05 \mathrm{~Hz}, n=7, p<0.01$; Fig. $2 B)$, suggesting that glycinergic synaptic transmission is indeed decreased in mutants. GlyT1 is known to remove glycine from the synaptic cleft, and the blockade of GlyT1 enhances glycinergic transmission
(Cui et al., 2005; Eulenburg et al., 2005; Mongeon et al., 2008). We therefore injected NFPS, an inhibitor of GlyT1, into the ventricles of mutant embryos. Although 28\% of NFPS-injected mutants (5/18 mutants) became immobile, $17 \%$ of the mu- 
tants (3/18 mutants) responded to touch without dorsal bend at the onset of the escape Movie 1, Clip 7). These results indicate that glycinergic synaptic transmission is compromised in nigl mutants.

To determine whether nig1 mutants have morphological defects at glycinergic synapses, the distribution of gephyrin, GlyRs, and acetyl tubulin was assessed by immunolabeling of spinal cord sections. Gephyrin and acetyl tubulin were found within the lateral region of the mutant spinal cord as in the wild type (Fig. 2C,D). Synaptic localization of GlyR clusters was also found at the lateral spinal cord in wild type but with lower intensity in mutants (Fig. 2E,F). Quantitative analysis confirmed that GlyR signals in mutant spinal cord was lower $(0.56 \pm 0.11, n=6, p<$ 0.05; Fig. $2 G)$ compared with wild-type zebrafish $(n=6)$. Western blotting of whole embryo protein extracts with antiGlyR $\alpha$ also showed that GlyR $\alpha$ subunit levels were decreased in mutants $(0.37 \pm$ $0.15, n=6, p<0.01$; Fig. $2 H, I)$ compared with wild-type zebrafish $(n=6)$, whereas the amount of gephyrin protein (1.06 $\pm 0.21, n=6)$ and acetyl tubulin protein $(1.10 \pm 0.14, n=6)$ was unchanged. These results, along with those of our behavioral assays and electrophysiology testing, indicate that glycinergic transmission is compromised due to a reduction of GlyR subunit levels in nig1 mutants.

\section{nig1 encodes the DEAH-box RNA helicase Dhx37}

We next performed genetic mapping of nig1 locus and identified a missense mutation (L489P) in an RNA helicase, DEAH-box protein 37 (Dhx37; GenBank accession number AB739007). To address whether the L489P mutation is responsible for $d h \times 37^{\text {nig } 1}$ mutant phenotype, we injected control (green fluorescent protein) RNA, wild-type Dhx37 RNA, or Dhx37 L489P RNA into recently fertilized embryos from $d h \times 37^{\text {nig1 }}$ heterozygous carriers and assayed touch responses. A quarter of control RNA-injected progeny $(29 \%, 22 / 75)$ and Dhx37 (L489P) RNA-injected progeny $(27 \%, 20 / 75)$ displayed the mutant response, whereas most of the progeny injected with wild-type Dhx37 RNA (94\%, 61/65) exhibited normal escape behavior, and only 6\% (4/65) displayed an abnormal response. The molecular identification of $d h \times 37$ as the causative gene was also supported by knockdown of Dhx37 using antisense MOs. Antisense Dhx37 MO1, which blocks translation of Dhx37, was injected into wild-type embryos. Most MO1-injected embryos (82\%, 71/87) displayed an abnormal touch response, whereas all control MO-injected embryos exhibited a normal response. Similarly, injection of Dhx37 MO2, which interferes with splicing of $d h \times 37$ mRNAs also induced a mutant phenotype $(91 \%, 84 / 92)$. These RNA rescue and antisense knockdown experiments confirm that the $d h \times 37^{\text {nigl }}$ mutation is responsible for the abnormal dorsal bend in the mutant escape behavior.
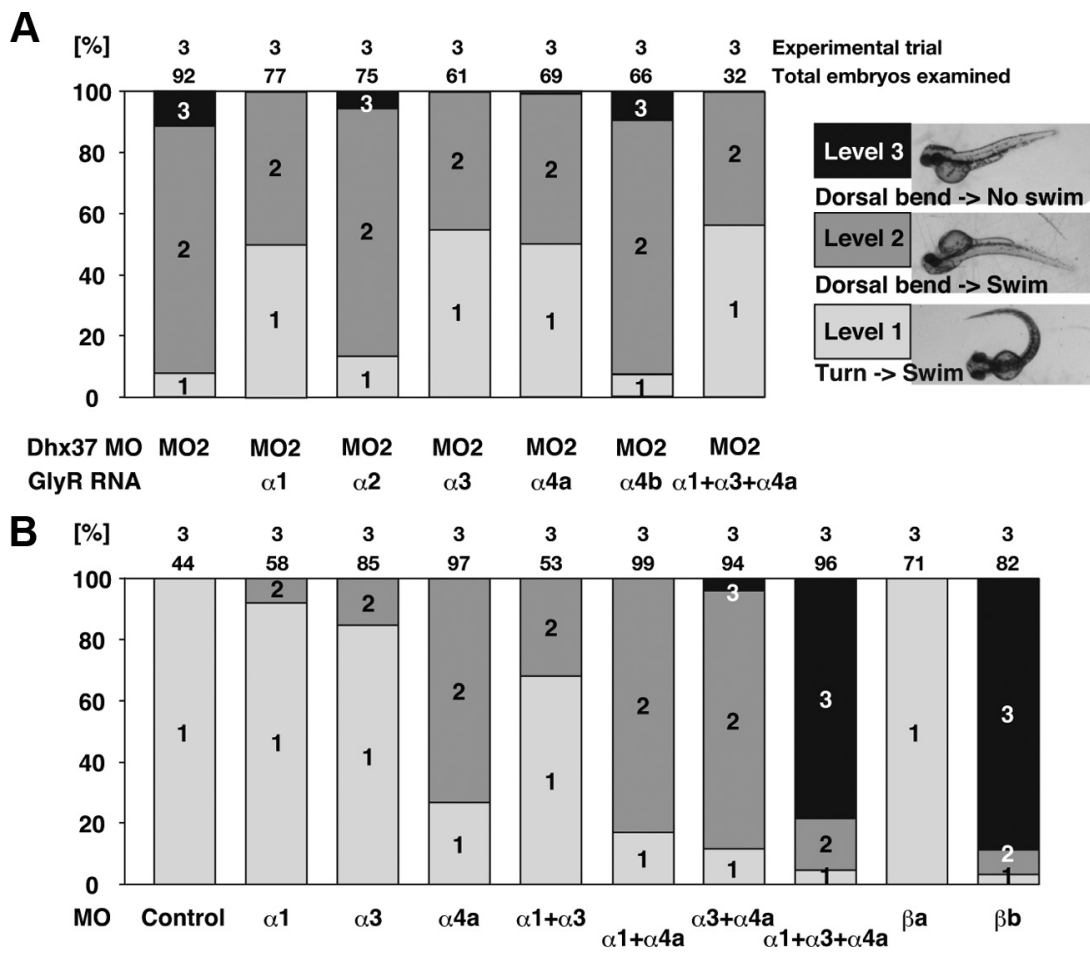

Figure 4. Expression of GlyR $\alpha 1, \alpha 3$, or $\alpha 4$ a subunits restored the normal touch response in Dhx37-deficient embryos. $\boldsymbol{A}$, njection of M02, which blocks the splicing of Dhx37, into wild-type embryos led to a dorsal bend response. Coinjection of either lyR $\alpha 1, \alpha 3$, or $\alpha 4$ a subunit RNAs with M02 decreased the abnormal behavior and restored the normal response. Mixed appliGlyR $\beta$ b M0-injected embryos displayed level 3 motor deficits.

\section{Dhx37 regulates biogenesis of GlyR $\alpha$ subunit mRNAs}

To investigate why a defect in the RNA helicase Dhx37 affects glycinergic synapses, we measured the expression of key genes involved in glycinergic transmission. These included five GlyR $\alpha$ subunits ( $\alpha 1, \alpha 2, \alpha 3, \alpha 4 \mathrm{a}$, and $\alpha 4 \mathrm{~b})$, two $\beta$ subunits ( $\beta \mathrm{a}$ and $\beta \mathrm{b}$ ), two isoforms of gephyrin ( $\mathrm{a}$ and $\mathrm{b}$ ), two glycine transporters (GlyT1 and GlyT2), and VIAAT. Our qPCR revealed that GlyR $\alpha 1, \alpha 3, \alpha 4 \mathrm{a}$, and $\beta \mathrm{a}$ subunits and gephyrin $\mathrm{b}$ transcripts were decreased in mutants $(\alpha 1: 0.74 \pm 0.09, n=6, p<0.05 ; \alpha 3$ : $0.74 \pm 0.08, n=6, p<0.05 ; \alpha 4 \mathrm{a}: 0.76 \pm 0.07, n=6, p<0.05$; $\beta \mathrm{a}: 0.62 \pm 0.05, n=6, p<0.01$; gephyrin b: $0.62 \pm 0.08, n=5$, $p<0.01$; Fig. $3 A$ ).

Some RNA helicases are involved in pre-mRNA splicing. Since qPCR can detect transcripts that are not fully spliced (i.e., could have a retained intron at another junction distant to the qPCR site), this method does not accurately measure the levels of fully spliced mRNAs. To quantify the amount of fully spliced mRNA, we performed Northern blotting and confirmed that GlyR $\alpha 1, \alpha 3$, and $\alpha 4$ a subunits and gephyrin b mRNAs were decreased in $d h \times 37^{\text {nigl }}$ mutants $(\alpha 1: 0.34 \pm 0.17, n=3, p<0.05$; $\alpha 3: 0.26 \pm 0.12, n=3, p<0.05 ; \alpha 4 \mathrm{a}: 0.24 \pm 0.11, n=3, p<0.01$; gephyrin b: $0.37 \pm 0.15, n=3, p<0.05$; Fig. $3 B$ ). The level of actin $\beta 1$ mRNA was unchanged in mutants $(1.03 \pm 0.14, n=3)$. We also tried to detect GlyR $\beta$ a mRNA, but specific bands were not detectable even in wild-type fish, presumably because the expression level is very low at this stage (Hirata et al., 2005). The amounts of $28 \mathrm{~S}$ and $18 \mathrm{~S}$ rRNA were comparable between wild- 
type and mutant fish (28S rRNA: $0.98 \pm 0.07, n=3$; $18 \mathrm{~S}$ rRNA: $1.04 \pm 0.13, n=3$ ). Our qPCR and Northern blot analyses indicate that mRNA levels for GlyR and gephyrin are decreased in $d h \times 37^{\text {nigl }}$ mutants, whereas rRNA biogenesis remains unchanged.

We next assessed the splicing of GlyR and gephyrin genes by RT-PCR, revealing that unspliced transcripts for the GlyR $\alpha 4 \mathrm{a}$ subunit are increased in $d h \times 37^{\text {nigl }}$ mutants (Fig. 3C). Similarly, unspliced gephyrin b products were upregulated. By contrast, splicing defects in actin $\beta 1$ transcripts were not observed in $d h \times 37^{\text {nigl }}$ mutants. We also attempted to detect unspliced transcripts for GlyR $\alpha 1$ and $\alpha 3$ subunit genes, but they were not accessible, because all of the introns are too long to amplify alongside normal short products. These results suggest that Dhx37 is involved in pre-mRNA splicing and that Dhx37 has substrate specificity for certain transcripts important for glycinergic synapse function.

The substrate specificity of Dhx37 was further investigated using an RNA immunoprecipitation assay. HEK293 cells were cotransfected with GlyR cDNAs that contained 5' - and 3'-UTR sequences together with a FLAG-tagged Dhx37 expression vector. Cell extracts were immuneprecipitated with anti-FLAG or with control IgG and subjected to RT-PCR. FLAG-Dhx37 immunoprecipitates contained GlyR $\alpha 1, \alpha 3$, and $\alpha 4$ a subunit transcripts, but not $\alpha 2$ subunit transcripts (Fig. 3D). Taken together, this assay clearly indicates that Dhx37 has substrate specificity for selected GlyR $\alpha$ subunit transcripts.

\section{Normal touch response is restored by overexpression of GlyR $\alpha$ subunits in $d h \times 37^{\text {nigl }}$ mutants}

Since RNA analysis revealed that the expression of GlyR $\alpha 1, \alpha 3$, $\alpha 4 \mathrm{a}$, and $\beta \mathrm{a}$ subunits and gephyrin b was decreased in $d h \times 37^{\text {nigl }}$ mutants, we investigated whether these GlyR subunits/gephyrin isoforms were also responsible for the behavioral deficiency. Capped RNAs for various GlyR subunits and gephyrin were synthesized in vitro and injected into wild-type embryos along with Dhx37 MO2 in three independent experimental trials. Injection of $\mathrm{MO} 2$ alone induced aberrant escape behavior, but coinjection of GlyR $\alpha 1, \alpha 3$, or $\alpha 4$ a subunit RNAs with MO2 increased the rate of normal behavior $(\alpha 1: 51 \%, 39 / 77 ; \alpha 3: 56 \%, 34 / 61 ; \alpha 4 a$ : $51 \%, 35 / 69$; Fig. 4A). Mixed application of GlyR $\alpha 1, \alpha 3$, and $\alpha 4$ a subunit RNAs with MO2 also restored the normal escape (56\%, $18 / 32)$. On the other hand, neither GlyR $\alpha 2$ nor $\alpha 4 \mathrm{~b}$ subunit RNAs restored the normal touch response ( $\alpha 2: 13 \%, 10 / 75 ; \alpha 4 \mathrm{~b}$ : $8 \%, 5 / 66$ showed normal responses). Likewise, embryos injected with MO2 and either GlyR $\beta$ a or $\beta$ b subunit RNA, or gephyrin a or b RNA failed to exhibit recovery of the touch response (data not shown). Thus, the shortage of GlyR $\alpha$ subunit, but not of GlyR $\beta$ or gephyrin, is responsible for the abnormal escape response in $d h \times 37^{\text {nigl }}$ mutants.

To assess which $\alpha$ subunit $(\alpha 1, \alpha 3$, or $\alpha 4 a)$ is primarily responsible for the GlyR $\alpha$ subunit depletion in mutants, we knocked down each GlyR subunit in wild-type embryos. Upon tactile stimulation, all control MO-injected embryos exhibited a lateral turn and swimming (Fig. 4B). However, injection of the GlyR $\alpha 1$ MO caused the mild level 2 phenotype in some fish (9\%, 5/58). Injection of the GlyR $\alpha 3 \mathrm{MO}$ increased the ratio of level 2 phenotype (15\%, 13/85), while the GlyR $\alpha 4 \mathrm{a}$ MO had a stronger effect in a greater percentage of fish $(71 \%, 69 / 97)$. Knockdown of the multiple GlyR $\alpha$ subunits had an additive effect-mixed application of two MOs decreased the touch response compared with single-MO injections $(\alpha 1+\alpha 3$ : 30.2\%, 16/53; $\alpha 1+\alpha 4$ a: $82 \%, 81 / 99 ; \alpha 3+\alpha 4 a$ : $88 \%, 83 / 94$ showed level 2 and 3 re- sponses). Moreover, the escape behavior of embryos injected with all three $\operatorname{MOs}(\alpha 1, \alpha 3$, and $\alpha 4$ a) was severely impaired (level 3 response, $76 \%, 48 / 63)$. Although $\beta$ a subunit morphants did not exhibit any behavioral defects, knockdown of GlyR $\beta$ b led to the severe level 3 response $(85 \%, 41 / 48)$, similar to the triple knockdown of $\alpha 1, \alpha 3$, and $\alpha 4$ a subunits.

\section{Discussion}

In summary, we have identified a zebrafish $d h \times 37^{\text {nigl }}$ mutant that displays an abnormal dorsal bend at the beginning of the escape response. GlyR $\alpha 1, \alpha 3$, and $\alpha 4$ a subunit mRNAs were downregulated and mis-spliced in $d h \times 37^{\text {nigl }}$ mutants. The depletion of GlyR $\alpha 1, \alpha 3$, and $\alpha 4$ a subunits is responsible for the motor deficits observed, since these could be improved by restoring expression of these GlyR $\alpha$ subunits. Mutations in human DHX37 may cause startle disease/hyperekplexia associated with defective glycinergic transmission.

\section{Substrate specificity of Dhx37}

The physiological significance of RNA helicases has been characterized in only a few cases. For example, mutations in the RNA helicase senataxin are responsible for neurodegenerative diseases (Moreira et al., 2004). Retinitis pigmentosa is caused by a dysfunction of pre-mRNA splicing factor 8 (Boon et al., 2007; Pena et al., 2007). However, the pathologically relevant substrate of these RNA helicases remains unknown. We demonstrated that Dhx37 interacts with selected GlyR $\alpha$ subunit mRNAs and specifically regulates biogenesis of these transcripts. Does Dhx37 have the other targets? Most embryos injected with $\mathrm{MO} 2$ and Dhx37 RNA displayed a normal touch response $(90 \%, 28 / 31)$, while embryos injected with $\mathrm{MO} 2$ and mixture of GlyR $\alpha 1, \alpha 3$, and $\alpha 4$ a subunit RNAs showed motor recovery at a lower efficiency $(56 \%, 18 / 32)$. This suggests that Dhx37 has targets other than GlyR subunit mRNAs.

\section{Subunit composition of GlyRs}

The initial characterization of purified GlyRs suggested that GlyRs were composed of $3 \alpha$ and $2 \beta$ subunits (Langosch et al., 1988), but recent functional and structural studies revised the stoichiometry to $2 \alpha 3 \beta$ (Grudzinska et al., 2005; Dutertre et al., 2012; Yang et al., 2012). Although our knockdown experiments indicate that $\alpha 4 \mathrm{a}$ appears to be the major GlyR $\alpha$ subunit involved in the escape behavior, the $\alpha 1$ and $\alpha 3$ subunits also contribute. We also showed that the $\beta$ b but not $\beta$ a subunit is essential for touch responses. This is consistent with our previous finding that the $\beta \mathrm{b}$ subunit is expressed by $1 \mathrm{dpf}$, while the expression of $\beta$ a is very low until $3 \mathrm{dpf}$ (Hirata et al., 2005). Taken together, it is likely that GlyR $\alpha 1, \alpha 3$, and $\alpha 4$ a subunits coassemble with the GlyR $\beta \mathrm{b}$ subunit, either in different receptor complexes or in heteromeric combinations, and that knockdown of all three $\alpha$ subunits is required to mimic the severe GlyR $\beta \mathrm{b}$-deficient phenotype.

\section{References}

Bleichert F, Baserga SJ (2007) The long unwinding road of RNA helicases. Mol Cell 27:339-352. CrossRef Medline

Boon KL, Grainger RJ, Ehsani P, Barrass JD, Auchynnikava T, Inglehearn CF, Beggs JD (2007) prp8 mutations that cause human retinitis pigmentosa lead to a U5 snRNP maturation defect in yeast. Nat Struct Mol Biol 14:1077-1083. CrossRef Medline

Colley A, Beggs JD, Tollervey D, Lafontaine DL (2000) Dhrlp, a putative DEAH-box RNA helicase, is associated with the box $\mathrm{C}+\mathrm{D}$ snoRNP U3. Mol Cell Biol 20:7238-7246. CrossRef Medline

Cui WW, Low SE, Hirata H, Saint-Amant L, Geisler R, Hume RI, Kuwada JY 
(2005) The zebrafish shocked gene encodes a glycine transporter and is essential for the function of early neural circuits in the CNS. J Neurosci 25:6610-6620. CrossRef Medline

Dutertre S, Drwal M, Laube B, Betz H (2012) Probing the pharmacological properties of distinct subunit interfaces within heteromeric glycine receptors reveals a functional betabeta agonist-binding site. J Neurochem 122: 38-47. CrossRef Medline

Eulenburg V, Armsen W, Betz H, Gomeza J (2005) Glycine transporters: essential regulators of neurotransmission. Trends Biochem Sci 30:325-333. CrossRef Medline

Fetcho JR, Higashijima S, McLean DL (2008) Zebrafish and motor control over the last decade. Brain Res Rev 57:86-93. CrossRef Medline

Granato M, van Eeden FJ, Schach U, Trowe T, Brand M, Furutani-Seiki M, Haffter P, Hammerschmidt M, Heisenberg CP, Jiang YJ, Kane DA, Kelsh RN, Mullins MC, Odenthal J, Nüsslein-Volhard C (1996) Genes controlling and mediating locomotion behavior of the zebrafish embryo and larva. Development 123:399-413. Medline

Grandi P, Rybin V, Bassler J, Petfalski E, Strauss D, Marzioch M, Schäfer T, Kuster B, Tschochner H, Tollervey D, Gavin AC, Hurt E (2002) 90 S pre-ribosomes include the $35 \mathrm{~S}$ pre-rRNA, the U3 snoRNP, and $40 \mathrm{~S}$ subunit processing factors but predominantly lack $60 \mathrm{~S}$ synthesis factors. Mol Cell 10:105-115. CrossRef Medline

Grillner S (2003) The motor infrastructure: from ion channels to neuronal networks. Nat Rev Neurosci 4:573-586. CrossRef Medline

Grudzinska J, Schemm R, Haeger S, Nicke A, Schmalzing G, Betz H, Laube B (2005) The beta subunit determines the ligand binding properties of synaptic glycine receptors. Neuron 45:727-739. CrossRef Medline

Hartman TR, Qian S, Bolinger C, Fernandez S, Schoenberg DR, Boris-Lawrie $\mathrm{K}$ (2006) RNA helicase A is necessary for translation of selected messenger RNAs. Nat Struct Mol Biol 13:509-516. CrossRef Medline

Hirata H, Saint-Amant L, Downes GB, Cui WW, Zhou W, Granato M, Ku- wada JY (2005) Zebrafish bandoneon mutants display behavioral defects due to a mutation in the glycine receptor beta-subunit. Proc Natl Acad Sci U S A 102:8345-8350. CrossRef Medline

Langosch D, Thomas L, Betz H (1988) Conserved quaternary structure of ligand-gated ion channels: the postsynaptic glycine receptor is a pentamer. Proc Natl Acad Sci U S A 85:7394-7398. CrossRef Medline

Lynch JW (2004) Molecular structure and function of the glycine receptor chloride channel. Physiol Rev 84:1051-1095. CrossRef Medline

McDearmid JR, Liao M, Drapeau P (2006) Glycine receptors regulate interneuron differentiation during spinal network development. Proc Natl Acad Sci U S A 103:9679-9684. CrossRef Medline

Mongeon R, Gleason MR, Masino MA, Fetcho JR, Mandel G, Brehm P, Dallman JE (2008) Synaptic homeostasis in a zebrafish glial glycine transporter mutant. J Neurophysiol 100:1716-1723. CrossRef Medline

Moreira MC, Klur S, Watanabe M, Németh AH, Le Ber I, Moniz JC, Tranchant C, Aubourg P, Tazir M, Schöls L, Pandolfo M, Schulz JB, Pouget J, Calvas P, Shizuka-Ikeda M, Shoji M, Tanaka M, Izatt L, Shaw CE, M'Zahem A, et al (2004) Senataxin, the ortholog of a yeast RNA helicase, is mutant in ataxia-ocular apraxia 2. Nat Genet 36:225-227. CrossRef Medline

Pena V, Liu S, Bujnicki JM, Lührmann R, Wahl MC (2007) Structure of a multipartite protein-protein interaction domain in splicing factor prp8 and its link to retinitis pigmentosa. Mol Cell 25:615-624. CrossRef Medline

Saint-Amant L, Drapeau P (1998) Time course of the development of motor behaviors in the zebrafish embryo. J Neurobiol 37:622-632. CrossRef Medline

Yang Z, Taran E, Webb TI, Lynch JW (2012) Stoichiometry and subunit arrangement of alphalbeta glycine receptors as determined by atomic force microscopy. Biochemistry 51:5229-5231. CrossRef Medline 